MaNY tumours produce proteins not normally made in any quantity by the tissue from which the tumour originates. This 'ectopic' production can result in hormones being synthesised in non-endocrine tumours or embryonic proteins being produced in adults. The most common explanation for ectopic protein synthesis is that it is a consequence of derepression of normally inaotive genes. While this is a possible explanation in some instances several observations are inconsistent with gene derepression as a general mechanism of ectopic protein production. First, direct measurements using nucleic acid hybridisation have failed to detect significant gene derepression in tumours and second, improved immunological techniques reveal the presence of ectopic proteins in normal tissue (see News and Views 269, 752; 1977). It seems that the most likely explanation for ectopic protein production is the increase in the abundance of mRNAs coding for ectopic proteins rather than new synthesis from previously inactive genes. A number of questions remain; what governs the amount of ectopic protein synthesis, why are certain ectopic proteins made by some tumours and not others and why does ectopic protein synthesis occur anyway?

It has been suggested that the amount of an ectopic protein produced by a cell is a function of its growth rate. There is for instance a positive correlation between $\alpha$ foetoprotein $(\alpha \mathrm{FP})$ production and growth rate in hepatomas; in rapidly growing regenerating liver $\alpha \mathrm{FP}$ levels are also elevated (Sell \& Becker J. natn. Cancer. Inst. 60, 19; 1978).

\title{
Ectopic hormone production by tumours
}

from Robert Shields

However, the relationship between growth rate and ectopic protein production is not simple, for instance, while human chorionic gonadotropin (hCG) production is high in fast growing and low in slow growing ovarian cystadenocarcinoma cells (Kanabus et al. Cancer Res. 38, 765; 1978) the reverse is true in HeLa cells (Ghosh et al. Biochem. J. 166, 265; 1977). While carcinoembryonic antigen (CEA) secretion is high in patients with inflammatory disease of colon and bronchus it is also high in slow growing bronchal carcinoma cells in culture (Ellison et al. J. natn. Cancer Inst. 59, 309; 1977). These subtle interrelationships between cell growth rate and protein synthesis are not confined to ectopic proteins, the amounts of various proteins made by hepatoma cells can be seen to vary widely under different growth conditions (Ivarie \& O'Farrell, Cell 13, $41 ; 1978)$. Perhaps it will be possible to make sense of these observations when we have a better understanding of the relationship between cell proliferation and protein synthesis.

Why a specific ectopic protein is often associated with a limited range of tumours and why only certain tumours produce ectopic hormones is not known. Attempts to answer these questions often claim that cancer represents some sort of dedifferentiation to a more embryonic form. For instance, almost all hepatocellular carcinomas and some pancreatic, gastric and pulmonary adenocarcinomas produce $\alpha \mathrm{FP}$. All these tumours are derived from tissue embryonically related to the yolk sac endoderm which is the embryonic site of $\alpha \mathrm{FP}$ synthesis. Dedifferentiation in these tumours is held to result in the reexpression of the ability to make $\alpha \mathrm{FP}$ (Sell \& Becker op. cit). This dedifferentiation hypothesis is also quite successful in explaining which tumours make CEA. A related embryological explanation for ectopic hormone synthesis is that all endocrine cells and those tumour cells secreting ectopic hormones share a common embryological origin in the neuroectoderm (Pearse \& Takor Takor, Clin. Endocr. 5, suppl., 229; 1976). According to this model hormone synthesis by a nonendocrine cell would represent a switch in gene function within a common embryonic lineage. While this idea goes some way towards explaining why some tumours and not others produce ectopic hormones there appear to be some exceptions (Rees Clin. Endocr. 5, suppl., 363; 1976). Of course this idea could be pushed to the ultimate extreme since in the final analysis all tissues in the adult derive from a common precursor, the fertilised egg.

Since so little is known about ectopic protein synthesis it may seem premature to ask why it occurs. There are probably a variety of reasons but a most intriguing possibility is that the production of ectopic protein is of benefit to the cell and has been selected for in the course of the evolution of the tumour. This will be discussed in a future article.

Robert Shields is at the Imperial Cancer Research Fund Laboratories, London. is simple, particularly in unhygienic surroundings: breast milk is nutritionally ideal, provides some immunity from disease, and is clean. Depending on the exact numerical values assigned to these two countervailing effects, the breast-to-bottle trend could increase or decrease net population growth.

\section{Overall patterns}

All the above factors may be drawn together, to arrive at the patterns illustrated in Fig. 2.

For the nomadic ! Kung, the relatively late age at menarche, 4 year birth interval, and relatively early age at menopause imply a completed family size of around five (three of whom would typically survive into their reproductive years).

Suppressing the temptation to air Views on their beliefs, I say only that the North American Hutterites are an
Anabaptist sect thought to be reproducing at close to the maximum rate under natural, breast-feeding conditions. The completed family size is around 11, and the population doubling time is a staggering 16 years. Note, however, that the relatively young age of onset of fertility is offset by social restraints that postpone intercourse and marriage until around age 22. Were Hutterites to marry at age 15 , there would be a slight increase in the completed family size, but a large increase in the growth rate (which is very sensitive to the age of first reproduction). If they put their minds to it, the Hutterites could possibly double their population every 10 years, thus solving a problem that troubled Sir William Petty in the nineteenth century, when he sought to prove the human population could have doubled every 10 years for a century after the
Biblical flood.

In a splendid review article, Frisch (op. cit.) has shown that patterns in mid-nineteenth century Britain were intermediate between the ! Kung and the Hutterites. The intermediacy embraces all facets: menarche; end of adolescent infertility; cultural infertility; birth spacing; and menopause. The average completed family size was around 6-8.

Against this background, the patterns (represented schematically in Fig. 2) for today's developed countries are qualitatively different.

\section{Contraception}

Short draws a significant message from the facts summarised in Fig. 2: "When we chose to develop artifical forms of contraception, we completely lost sight of the normal reproductive life-history of our species". 